\title{
Content-Based Image Retrieval of Color, Shape, and Texture by Using Novel multi-SVM Classifier
}

\author{
Mudhafar J. J. Ghrabat, Guangzhi Ma, Paula Leticia Pinon Avila, Muna J. Jassim, and Safa J. Jassim
}

\begin{abstract}
Background: the considerable time consumption, query retrieval difficulty and reduced retrieval rate. Still remaining challenges in Content-based image retrieval.

Methods: in this work, we propose a pre-processing method that uses a Gaussian filter to improve quality by reducing image noise. An effective feature extraction method for in presented to extracted texture help color co-occurrence feature (CCF), color and shape features such as area and diameters. The colors features are extracted by means of a grey-level co-occurrence matrix and bit pattern. Extracting these features will enhance the image retrieval accuracy. With the use of a novel multi-SVM classifier, classification is performed and image retrieval is completed effectively.
\end{abstract}

Results: performance measures, namely, precision, recall, error rate, correct rate, and retrieval rate, are computed. The proposed methodology produces superior results on these measures and exhibits an effective retrieval rate of

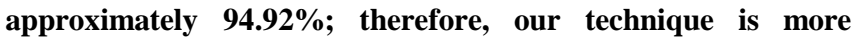
efficient than existing MRED and MALP methods.

Index Terms-Pre-processing, feature extraction, novel Multi-SVM classifier, color co-occurrence feature (CCF), grey level co-occurrence feature (GLCM), bit pattern feature (BPF).

\section{INTRODUCTION}

Image retrieval is a fast growing and stimulating research area is image processing, which is widely applied in computer vision recognition, pattern recognition, object visualization, and texture recognition. The major problem faced by the image retrieval is mismatch errors that are caused by the extraction of similar images related to the query. The manual indexing of textual data is called text- or concept-based image indexing. Whose performance, relies on the

Proficiency of the feature extraction and the exact retrieval of identical images. The content-based image retrieval (CBIR)

Manuscript received February 12, 2019; revised June 1, 2019. This study is done in the Chinese Education Ministry Key Lab of Image Processing and Intelligent Control, and the Centre for Biometrics Research in Southern Polytechnic State University in United States. It is funded and supported partly by grant 61075010 for the project "Research on Non-rigid Registration Method for Multi-model Cardiac Images Based on Different Direction Features" of the Natural Science Foundation of China. This work is also supported in part by grant 2012AA02A609 for the Chinese National 863 Project "Digitalized Traditional Chinese Medicine Information System Research and Development.

Mudhafar J. Alghrabat, Guangzhi Ma, Paula Leticia Pinon Avila are with Huazhong University of Science and Technology, Wuhan, China (e-mail: mudhafarjalil@hust.edu.cn, paulapiav@ hotmail.com).

Muna Jalil Jassim was with the Alrafidain University College, School Computer Engineering, Baghdad, Iraq (e-mail: munam3040@gmail.com).

Safa J. Jassim is with the Electrical Engineering Department, Al-Mustansiriya University, Baghdad, Iraq (e-mail: engsafa23@gmail.com). can be determined [1] by their texture, color and shape attributes. The color is deliberated as a significant dispute [2] not only insight but also in texture. Its impact is still elevating in the area of digital image processing. Colour is a directional feature that contradicts the intensity coded by scalar grey standards.

One general belief is that the more feature descriptors are present, the better the image recognition performance. However, not all features are very useful in image acknowledgment. The ill features of interfering signals usually lead to decreased recognition rates. DBPSP and the color co-occurrence matrix (CCM) are typically used for identifying links between textures and [3] colors, and CHKM is utilized for retrieving image color information. Meanwhile, image integration is useful in handling pictures retrieved from enormous datasets. Different images involve various low-quality pictures, such as cartoon, natural, grey-textured and colored, categorized and textured images. The system performance is degraded as the response time increases when retrieval image from massing dataset. Searching a massive dataset requires sophisticated searching skills. Usually, annotations are manually provided for images to describe a set of keywords, this process is time-consuming and thus calls for the use of CBIR.

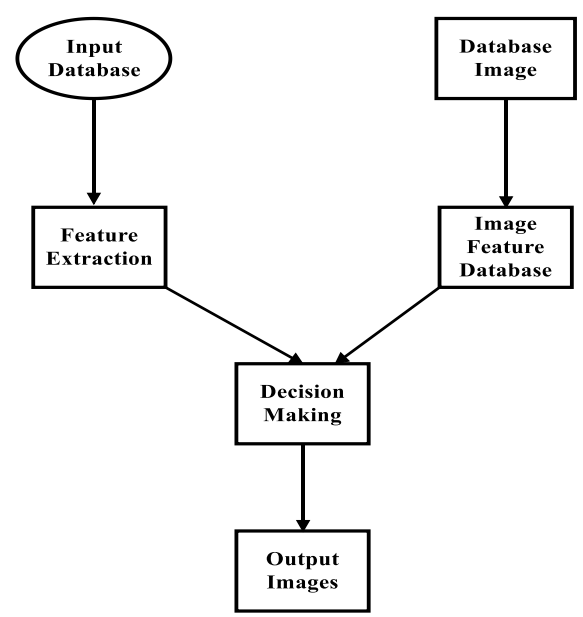

Fig. 1. Content-based image retrieval.

The use of existing SVM classifier has some limitations such as minimized accuracy, sensitivity, and precision. Thus, the novel multi-SVM (NMSVM) classifier is proposed in this research, which enhances the better performance in precision, recall, specificity, accuracy and sensitivity measures. In this work, image features are observed as the directory of the image, and retrieval retrieved input query image is accomplished on that particular image. A technique of CBIR is applied for exploiting the advantages of small complication index color images to identify the redundant color pixels 
index using the extracted features. The texture color and shape information is considered the primitive descriptors of the image. To attain high efficiency, these descriptors are considered together along with the image and its complement. Afterward, with the use of test and train values, the features are extracted and the classifier is utilized for the retrieval method. The key objectives of this work are as follows:

a) To differentiate query images from the database images.

b) To retrieve images from large databases by use of CBIR, which depends on texture, color, and other attributes.

c) To increase the retrieval rate and reduce retrieval time.

The processes in our work involving shape and color in this work are as follows:

a) We introduce a novel multi-SVM classifier extracted features for the retrieval of images similar to the provided query.

b) The color co-occurrence feature $(\mathrm{CCF})$ is adopted for color extraction. Area and diameter are utilized for shape extraction. GLCM and BPF are used for texture extraction.

c) Finally, we measure the performance of the retrieval process.

The main contribution of this work is as follows:

a) The accuracy and the retrieval rate of the challenge are less hence and texture feature for the extraction process, the accuracy of the method can be improved.

b) The limitations of the proposed methods are then overcome by using this efficient feature extraction method and a novel Multi-SVM classifier for the process of classification.

The remainder of this paper is organized as follows. Section II reviews works related to several conventional CBIR methods in image processing. Section III introduces the proposed CBIR-NMSVM methodology for image retrieval and classification. In Section IV, we evaluate the performance of the proposed mechanism, and Section $\mathrm{V}$ presents the conclusion.

By using the novel multi-SVM classifier the detection accuracy can be increased on comparing to Multi-SVM classifier. Thus the images that are appropriate can be retrieved more accurately.

\section{RELATED WORKS}

A previous study presented and estimated the retrieval of interactive similar images [4] in relation to the similarity of color images, such as texture or color features measure. Feature extraction and matching operations were performed in image retrieval. Of [5] the first process involved extracting features from a range of distinguishable ones, and the second included matching similar images to obtain improved outcomes. The semantic gap between the results of image similarity and user perception are the defects of this effort.

The ordered-dither block truncation coding, a feature extraction method [6] that applies half toning-dependent block truncation coding, was presented to remove the semantic gap between the truncated coding. This method generated descriptors image content. Block truncation coding involves condensing images and requires a simple procedure that comprises decoding and encoding stages. This method yielded a better precision in image retrieval than state-of-the-art techniques did. However, this scheme cannot bridge the gap between explicit semantic knowledge, image content, and there are subjective criteria for assessment and human-oriented testing.

The grid-based indexing was presented [7] for retrieving images with high accuracy. This method allows algorithms to avoid signal and noise distortion during processing but encounters difficulties in addressing the definite size of feature vectors.

A novel [8] survey was performed to overcome the challenge of reducing the semantic gap between the high-level semantics of images and the low-level features of visual parameters. In this method, the image of query features is associated with image database features using a measure of distance. An analysis in terms of prediction of recognition rate, precision, and recall analysis measures. Comparison in this study showed that the survey method's capability of recognizing image semantics has been enhanced.

The new semantic [9] features were abstracted to overcome the difficulty of certain dominant color descriptors to reduce the effect of background images. This reduction is accomplished via image matching decision, in which the object that is to be retrieved receives increased focus. The benefit of this methodology is its good semantic feature extraction performance on similarity measures. However, the efficacy of the similarity measure needs improvement. An innovative method was developed for CBIR using the graph-based model of ranking [10]. This method limits the geometrical configuration of the underlying image database provided. The main advantage of this methodology is its capability to overcome the limits of other methods, such as scalable construction of graphs and ranking computation, and become more effective. The algorithm of effectual assorted classifier performed effectively for large dataset. Although in handling vast databases and decreases the computation time, the accuracy is not high. A comparison and survey between the HSV and RGB methods [11] were performed. The basis of precision, accuracy, and recall. In this method, a similarity algorithm is utilized to determine the similarity degree between two images. This survey showed that the existing image retrieval system had certain benefits and shortcomings regarding text- and image-based queries. Combing these two retrieval techniques is essential to improve the value of image retrieval systems. A study [12] explored the use of hashing-based image retrieval. This approach uses CBIR and computer-aided diagnosis for disease detection, diagnosis and decision support. This technique is effective in calculation and storage but may fail to incorporate the feature fusion mechanism into the hybrid hashing methodology, which may improve image retrieval precision.

An innovative study presented [13] an unsupervised visual hashing method known as semantic-assisted visual hashing. This approach was utilized to associated modalities involved. CBIR via sketching was presented [14]. In this technique, images are annotated manually to previse of keywords for 
retrieving image via text-based search. This approach matched legal sketches and mug shots from an image gallery but failed to discriminate the unaltered images from color images or edge representations. CBIR retrieval method was used [15] for extracting images that were similar in feature as query images from image databases. This method is practical in crime prevention, face detection, image fusion, and digital libraries. At the digital libraries, it can retrieve the relevant books name, novel name and author name but lacks effectiveness in certain situations. A technique that adopts visual enhance the aptitude of visual hashing without explicit semantic labels but failed exhibit effectiveness when additional contents CBIR [16] such as features for querying images from large-scale datasets was implemented for it. For industrial and commercial uses, texture information can be developed to improve method accuracy, possibly enhancing the color extent of images. The threshold parameters must be fine-tuned and feedback should be offered to overcome disadvantages and realize improvement. A CBIR technique [17] that uses shape, color and feedback relevance was utilized for the correct representation of color and shape in a semantic manner. The proposed methodology deals with the shape descriptor, which must be robust against geometric attacks to images. However, the technique does not provide efficiency in relevant information efficiency meant for accurate relevant information.

A comparison between [18] the features of shape and combination texture was performed utilizing GLCM and $\mathrm{Hu}$ moments. In this method, recall and precision are the most generally used performance estimation parameters. Although the system is implemented well by this combination, the consequence must be pre-processed during retrieval.

A novel CBIR technique that abstracts each image in a database by [19] computing statistical features with the use of a multi-scale geometric study of the non-sub-sampled contourlet transform was proposed. The relevance feedback appliance was incorporated into the graph-theoretic approach. The most noteworthy enhancement of this methodology is noise suppression. However, sequential measurement is not effectively exploited in this methodology.

CBIR methods are demanded [20] in several applications, such as data mining, education, weather forecasting, medical imaging, earth resource management, and remote sensing.

According to the above review of existing approaches, the following are the major disadvantages in image retrieval.

a) The computation of ranking is insufficiently accurate and effective.

b) The accuracy is poor in terms of retrieval rate.

c) Precision value is low.

d) The features that extracted from images are insufficiently accurate.

e) Existing methods' retrieval rate of similar images regarding query images has low relevant.

f) Classification of extracted features yields poor accuracy. The proposed method solves the aforementioned issues through the following means.

a) A pre-processing technique is applied for the removal of noise from images, thereby enhancing image quality. b) Features such as color, shape, and texture are extracted using effective methods, such as CCF, area and diameter, GLCM and BPF, hence increasing system accuracy.

c) Classification is conducted by using a novel trained classifier (NMSVM), thus increasing classification accuracy.

d) Finally, the retrieval process improves the retrieval rate of images similar to the query.

\section{PROPOSED WORK}

This section explains the implementation of image retrieval and classification for images in a database. Images are initially extracted from dataset, and then a pre-processing method that adopts a Gaussian filter is used to filter the acquired images. The feature extraction procedure is then used for extracting textures, shape, and colors. In this feature extraction technique, the color feature is extracted with the aid of CCFs, the shape feature is extracted using area and diameters and the texture feature is extracted through GLCM and BPFs. After extraction, the NMSVM classifier is train and text features with label and text features on input. The retrieval process is then performed from the classified image, for which the performance measures, namely, precision, recall, retrieval rate, correct rate and error rate, are estimated. The idea in our work use many methods such as pre-processing, feature extraction and retrieval process are introduced in this paper. As the flow of this work is like that it can be organized as it is to make the retrieval process an effective retrieval rate and correct the rate error.

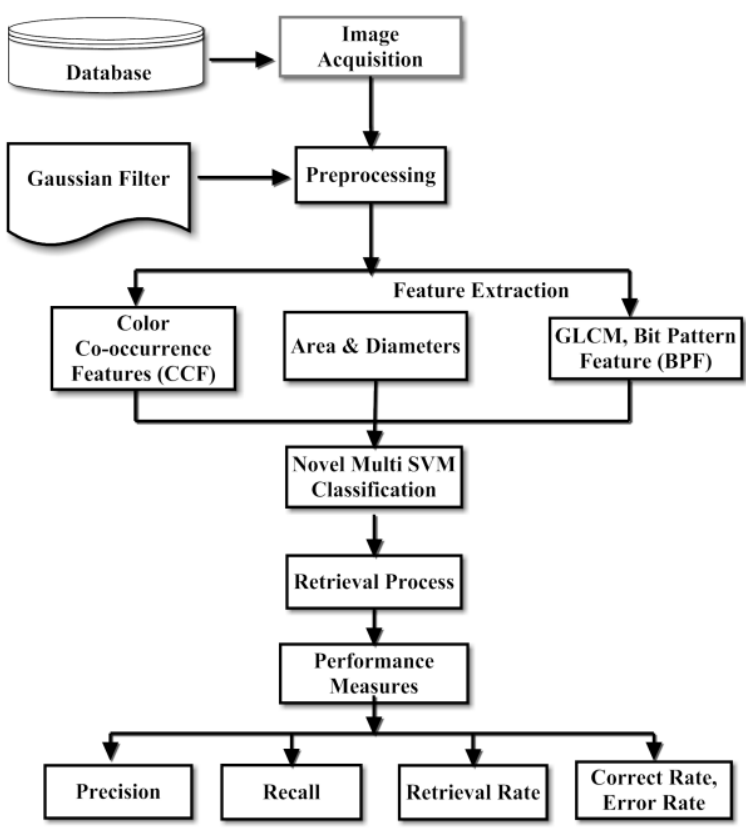

Fig. 2. The overall flow of the proposed system.

\section{A. Pre-processing}

Pre-processing is essential in image processing or retrieval. Input images are extracted from the database and then pre-processed to boost image efficiency. In this stage, the image features are enriched by the application of a Gaussian filter, which is usually utilized to decrease the noise as well as the presence of blur in images. Figure 3 shown in this process 
eliminates the image noise and reduces the image dimension. The use of this filter is also helpful edge detection. The Gaussian filter function is expressed as

$$
G(x, y)=\frac{1}{2 \pi \sigma^{2}} e^{-\frac{x^{2}+y^{2}}{2 \sigma^{2}}},
$$

where

' $\sigma$ ' is the distribution's standard deviation.

where $x$ is the distance from the origin along the horizontal axis and $y$ is the distance from the origin along the vertical axis.
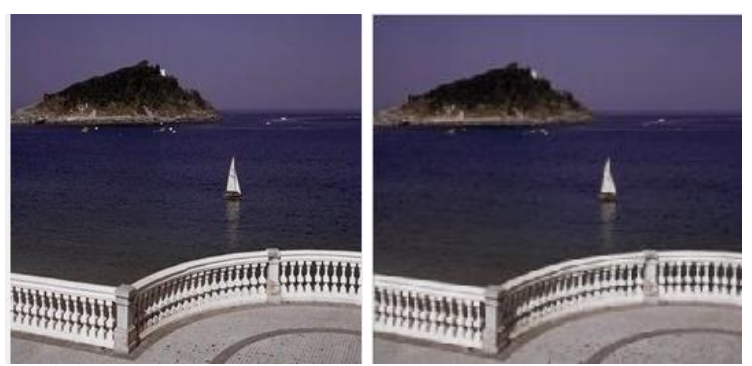

(a) Input image (b) Gaussian filtered image Fig. 3. Pre-processed image.

\section{B. Feature Extraction}

Feature extraction is the transfer of the input data into the set of features, separating the accurate data from the vast set of databases and the recovery of the appropriate and revealing features (also known as dimensionality reduction). In this stage, image features (shape, texture, and color) are extracted. The idea to make steps are introduced. These three are important features in the process of feature extraction.

\section{1) Colour feature}

\section{a) Colour co-occurrence feature (CCF)}

The massive quantity of information about the image content is presented in the color distribution of pixels in the image. The distribution of image color provides the attributes of that image with the support of CCM. This type of matrix is used to compute the pixel's probability of occurrence together with the nearby pixels for the determination of constructing the information of the desired color Table I the results of color features.

\begin{tabular}{cc} 
TABLE I: SIMULATION OUTPUT OF COLOUR FEATURE \\
\hline \hline Serial. no & Colour Feature \\
\hline 1 & 2.625 \\
2 & 2.9375 \\
3 & 2.4375 \\
4 & 1.125 \\
5 & 0.6875 \\
6 & 0.25 \\
7 & 0.25 \\
8 & 0 \\
9 & 0.0625 \\
10 & 0 \\
\hline \hline
\end{tabular}

\section{2) Shape feature}

This feature is extracted from images with regard to its shape. Shape feature extraction adopts the area and diameters, as shown in Table II.

The area is signified in the actual amount of white pixels in the selected object region. The object area is computed by the sum of white or ' 1 ' pixels inside the object boundary. Diameter is the measure of summation of the distances between each neighboring pair of pixels throughout the object edges.

TABLE II: SIMULATION OUTPUT OF SHAPE FEATURE
\begin{tabular}{cc} 
S. No & Shape Feature \\
\hline 1 & 65536 \\
2 & 3779.682 \\
\hline
\end{tabular}

\section{3) Texture feature}

Texture retrieval for feature extraction requires the following:

- Grey level co-occurrence matrix (GLCM)

- Bit pattern features (BPF)

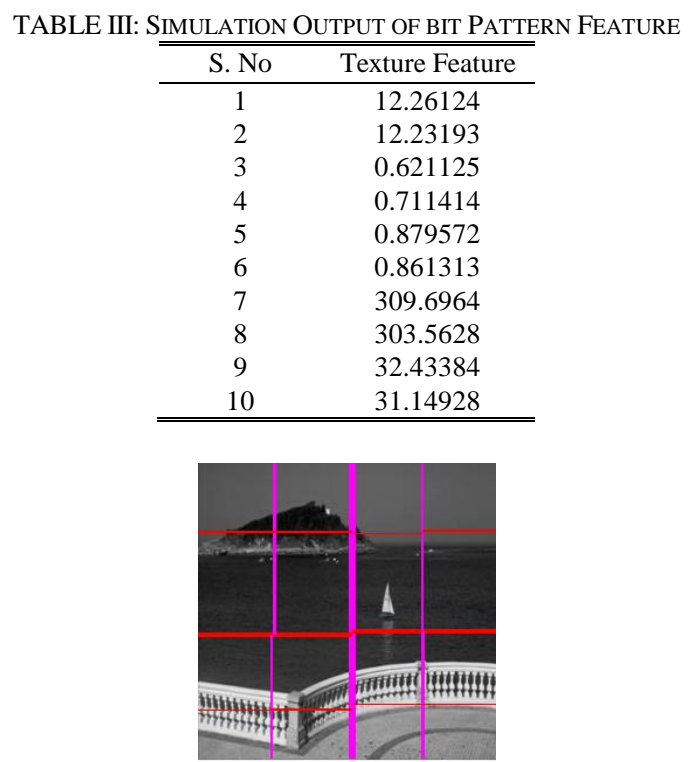

Fig. 4. Bit pattern feature image.

\section{Retrieval Process}

The multi-SVM classifier is used in the sorting of the relevant and irrelevant images from a massive dataset. For the two separate classes, the manner of a grouping of these two classes is well-defined via multi-SVM. The training of the SVM classifier at the root node of a decision tree is performed by this kind of classification, in which the samples obtained from the first type is deemed accurate and the one obtained from the other class is considered incorrect. Studies about various multi-SVM classifiers have shown that the accuracy, precision and retrieval rates of such classifiers are not satisfactory and is a required improvement. Similar images are classified whilst different images are separated by this classifier. In the present work, the probabilistic neural network PNN is incorporated into the multi-SVM, thereby forming the NMSVM classifier. In this combination, the multi-SVM classifier utilizes the label related features to train and test the processed image and to estimate the performance measures. The use of the NMSVM classifier enhances efficiency by improving the retrieval rate. At the same time, the rate of accuracy and precision also increases.

The NMSVM classifier is used in this work, in which the input is given with the label and its related features. In the 
initial step, the image features are obtained and their size and value are computed, and this new train and test datasets are constructed according to these extracted features, where $[\mathrm{m}, \mathrm{n}]$ represents the rows and columns of the matrix. Afterward, the class labels are identified, in and the unique classes are found in each train and test dataset. Therefore, is determined by the number of unique classes. In the next step, the SVM structure is computed and the output for the unique class is converting to a vector form. Trainor is applied to identify the minimum and maximum classes. Then, the train datasets are integrated with the new Probabilistic Neural Network PNN. Subsequently, similar images are retrieved from 100 related images.

The proposed method is efficient in retrieving the data that are similar to the query image provided as shown in Fig. 5. This efficiency is the main advantage of this work.

\section{ALGORITHM: NOVEL MULTI-SVM CLASSIFIER}

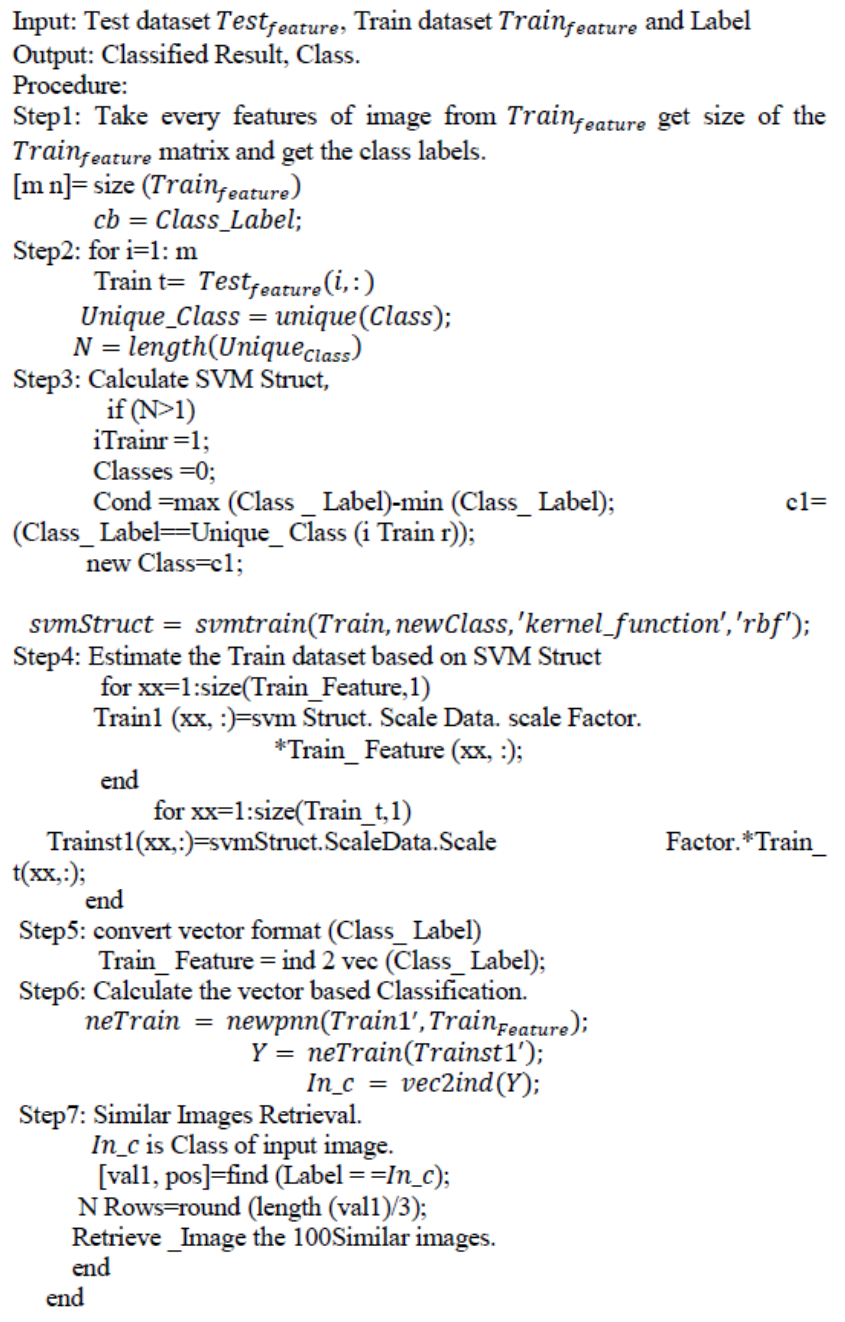

The integration of multi-SVM with PNN allows the acquisition of results with improved precision. The conversion into vector form leads to retrieval good rate.

Fig. 5 depicts similar images retrieved according to the query provided. A class, which consists of 100 images, is multiplied by 10 classes. Hence, 1000 images are present in total and comprise the core $1 \mathrm{k}$ dataset which is taken in this method. Fig. 6 represents in the form of a $10 \times 10$ matrix.

The test, label and train features were used in the NMSVM classifier. The simulation output is as follows.

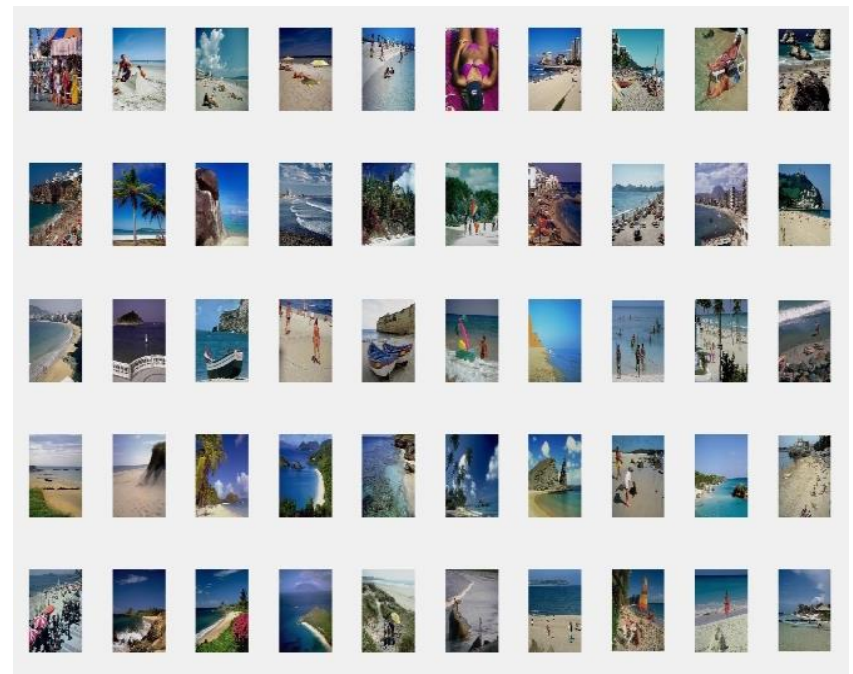

Fig. 5. Similar query images.

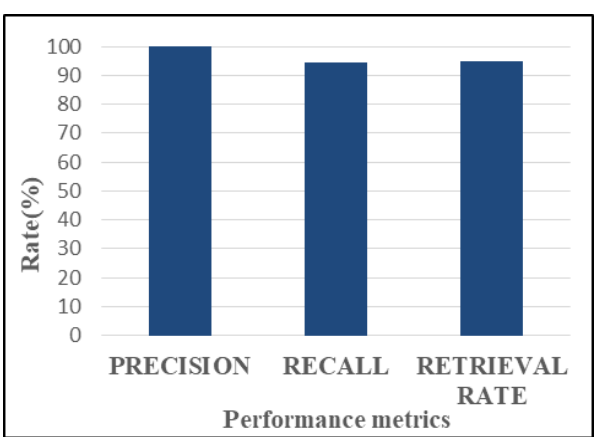

Fig. 6. The performance measure of precision, recall, and F-measure.

\begin{tabular}{cr} 
TABLE VI: SIMULATION RESUlt OF TEST FEATURE \\
\hline S. No & \multicolumn{2}{c}{ Test Datasets } \\
\hline 1 & 2.625 \\
2 & 2.9375 \\
3 & 2.4375 \\
4 & 1.125 \\
5 & 0.6875 \\
6 & 0.25 \\
7 & 0.25 \\
8 & 0.0625 \\
9 & 65536 \\
10 & 3779.682 \\
\hline \hline
\end{tabular}

The simulation output of the train feature is obtained in the form of a dataset depicted in Table VII.

\section{PERFORMANCE ANALYSIS}

The performance metrics are evaluated using MATLAB (R2017b). The performance of the NMSVM classifier is evaluated in this section.

\section{A. Dataset Description}

In the implementation of this method, the Corel $1 \mathrm{k}$ dataset is utilized. This research uses the commercially accessible Corel photograph gallery, which contains 1000color images with $384 \times 256$ pixels. All images in the database are grouped into 10 classes, each class involves 100 images in each with several semantic groups, such as 'beach', 'building', 'people', 'elephant', 'dinosaur', 'flower', 'mountain', 'food', 'horse' and 'bus'. Pictures belonging to the same class or semantic group are considered similar images. 


\section{B. Performance Metrics}

The performance of the proposed NMSVM approach is demonstrated in this section in relation to the following metrics: sensitivity, specificity, precision, recall, and accuracy, which are evaluated with the novel classifier that is used along with the integration of Probabilistic Neural Network PNN.

TABLE VII: SIMULATION OUTPUT OF TRAIN FEATURE

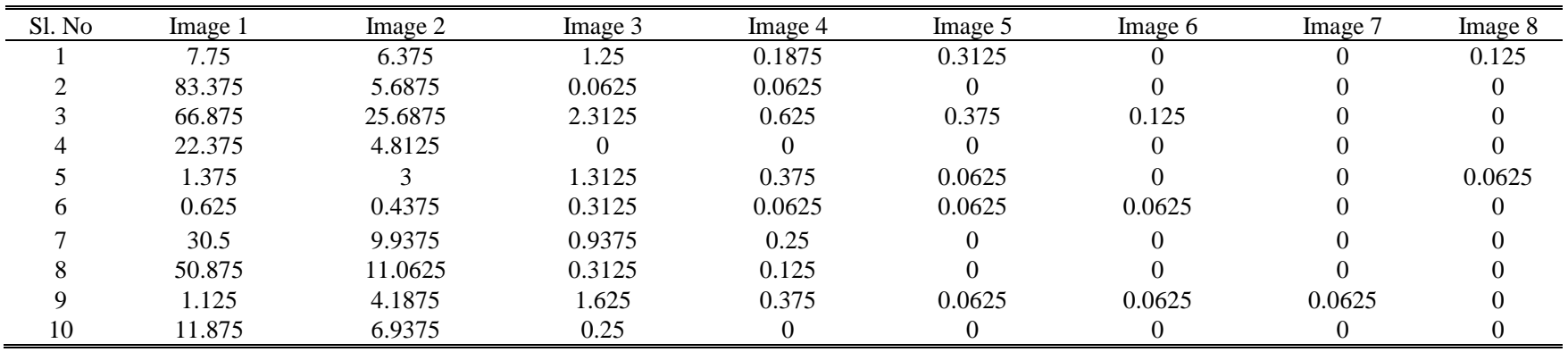

\section{Accuracy, Precision and Recall Analysis}

An analysis of precision, accuracy, and recall is conducted for the NMSVM approach, and the results are compared with those obtained from existing methods.

Accuracy: Accuracy is defined as the intimacy of a restrained rate to the average values. It is named as the load arithmetic mean of precision and reverse precision.

$$
\begin{aligned}
& \text { Accuracy }=\frac{T P+T N}{T P+T N+F P+F N}= \\
& \frac{\text { (Number correctassessments) }}{\text { (Number of all all assessments) }}
\end{aligned}
$$

Sensitivity: The sensitivity is labeled as true positive and utilized to compute the ratio of positive that are suitably recognized features in the image.

$$
\begin{aligned}
& \text { Sensitivity }=\frac{T P}{T P+F N}= \\
& \frac{\text { (Number of true positive assessments) }}{\text { (Number of all all positive assessments) }}
\end{aligned}
$$

Precision: Precision and recall are simple parameters that are generally utilized to demonstrate the classification method. In image retrieval, precision is the division of recovered images that are significant to query images. It is expressed as

$$
\text { Precision }=\frac{T P}{(T P+F P)} * 100
$$

Recall: Recall measures the calculation prototype's capability that is mostly utilized to choose the rate of a definite class from a dataset. It is similarly known as sensitivity, which is computed as

$$
\text { Recall }=\frac{T P}{(T P+F N)} * 100
$$

Specificity: Specificity is the measure of the ratio of actual negatives, which are accurately identified. It is written as

$$
\begin{aligned}
& \text { Specificity }=\frac{T N}{(T N+F P)}= \\
& \frac{\text { (Number of true negative assessment) }}{\text { (Number of all negative assessment) }}
\end{aligned}
$$

The performance measure is also estimated for precision, F-measure, and recall. The proposed method yields a precision value of $100 \%$, recall value of $94.444 \%$ and retrieval rate value of approximately $94.90 \%$. These performance metrics are graphically depicted below.

\section{COMPARATIVE Results}

A comparative analysis is performed for existing methods (MRED and MALP) and the proposed technique (NMSVM classifier) in CBIR. The precision, recall and retrieval rate values are estimated, and the proposed methodology produces higher values than do the extant techniques. The proposed methodology performs well, yielding a precision value of approximately $100 \%$, recall value of 94.444 and retrieval rate of $94.9 \%$. A comparison of these values with those of the existing techniques reveals that the efficiency of the system is improved.

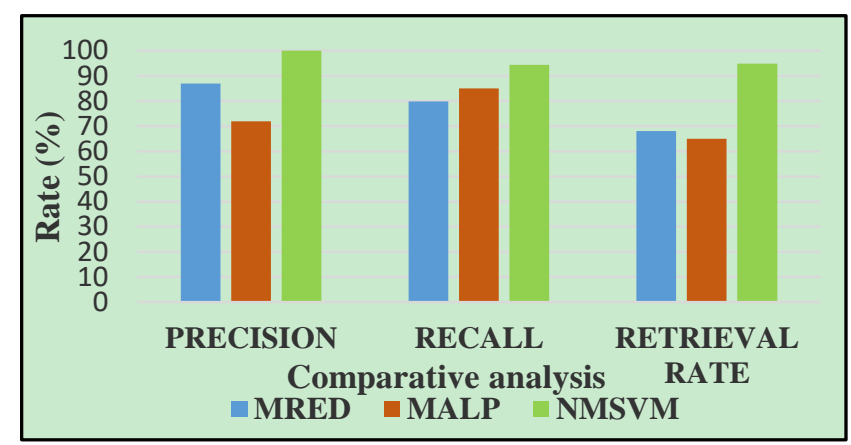

Fig .7. Comparative analysis.

TABLE VIII: COMPARATIVE ANALYSIS OF VARIOUS ALGORITHMS

\begin{tabular}{llll}
\hline \hline Techniques & PRECISION & RECALL & RETRIEVAL RATE \\
\hline MRED & 87 & 79.9 & 68 \\
MALP & 72 & 85 & 65 \\
NMSVM & 100 & 94.444 & 94.9 \\
\hline \hline
\end{tabular}

\section{CONCLUSION}

Image retrieval is the process of retrieving an appropriate image that is appropriate to an input provided from a vast database. CBIR is the content-based image retrieval process where the content may be an image, text, etc. according to the input. In this work, CBIR is implemented by the user (NMSVM) classifier. The incorporation of the multi SVM classifier with PNN is proposed. Initially, the Gaussian filter is employed for the removal of salt and pepper noise in the 
pre-processing stage. Feature extraction is very good performed due to the use of Multi-SVM incorporated with PNN (NMSVM), and the images are grouped with the classifier. Finally, the images are retrieved. With these retrieved images, performance measures are calculated in terms of retrieval rate, precision, recall, error rate, and correct rate. The proposed technique yields a retrieval rate of $94.90 \%$, the precision of $100 \%$, and recall of approximately $94.444 \%$, which are higher than existing methods of MRED and MALP.

\section{CONFLICTS OF INTEREST}

The authors declare that there are no conflicts of interest regarding the publication of this paper.

\section{ACKNOWLEDGMENT}

This study is done in the Chinese Education Ministry Key Lab of Image Processing and Intelligent Control, and the Centre for Biometrics Research at Southern Polytechnic State University in the United States. It is funded and supported partly by grant 61075010 for the project "Research on Non-rigid Registration Method for Multi-model Cardiac Images Based on Different Direction Features" of the Natural Science Foundation of China. This work is also supported in part by grant 2012AA02A609 for the Chinese National 863 Project "Digitalized Traditional Chinese Medicine Information System Research and Development".

\section{REFERENCES}

[1] D. S. Shete, M. Chavan, and K. Kolhapur, "Content-based image retrieval," International Journal of Emerging Technology and Advanced Engineering, vol. 2, pp. 85-90, 2012.

[2] C. Palm, "Color texture classification by integrative co-occurrence matrices," Pattern recognition, vol. 37, pp. 965-976, 2004.

[3] C.-H. Lin, R.-T. Chen, and Y.-K. Chan, "A smart content-based image retrieval system based on color and texture feature," Image and Vision Computing, vol. 27, pp. 658-665, 2009.

[4] M. Rehman, M. Iqbal, M. Sharif, and M. Raza, "Content-based image retrieval: Survey," World Applied Sciences Journal, vol. 19, pp. 404-412, 2012.

[5] G. Ashutosh, and M..Gangadharappa, " March. image retrieval based on color, shape, and texture, " in Proc. 2015 2nd International Conference on Computing for Sustainable Global Development, pp. 2097-2104.

[6] B. Mounika, Y. Sowmya, S. Pasala, and A. Sravani, "Content-based image retrieval using color," International Journal of Applied Engineering Research, vol. 11, pp. 4331-4334, 2016.

[7] F. Rajam and S. Valli, "A survey on content-based image retrieval," Life Science Journal, vol. 10, pp. 2475-2487, 2013.

[8] A. Talib, M. Mahmuddin, H. Husni, and L. E. George, "A weighted dominant color descriptor for content-based image retrieval," Journal of Visual Communication and Image Representation, vol. 24, pp. 345-360, 2013

[9] B. Xu, J. Bu, C. Chen, C. Wang, D. Cai, and X. He, "EMR: A scalable graph-based ranking model for content-based image retrieval," IEEE Transactions on Knowledge and Data Engineering, vol. 27, pp. $102-114,2015$

[10] S. Kaur and D. V. K. Banga, "Content-based image retrieval: Survey and comparison between the RGB HSV model," International Journal of Engineering Trends and Technology, vol. 4, pp. 575-579, 2013.

[11] X. Zhang, W. Liu, M. Dundar, S. Badve, and S. Zhang, "Towards large-scale histopathological image analysis: Hashing-based image retrieval," IEEE Transactions on Medical Imaging, vol. 34, pp. 496-506, 2015

[12] L. Zhu, J. Shen, L. Xie, and Z. Cheng, "Unsupervised visual hashing with a semantic assistant for content-based image retrieval," IEEE
Transactions on Knowledge and Data Engineering, vol. 29, pp. 472-486, 2017.

[13] S. Parveen, S. Yadav, and N. Chauhan, "Sketch4Match-content-based image retrieval system using sketches," International Journal (Toronto, Ont.), vol. 3, 2013

[14] A. Tewari, U. Nikhat, and S. Malik, "Content-based Image Retrieval," International Journal of Computer Applications, 2015.

[15] A. K.Shukla, S. Kanungo, "Content-Based Analysis of Biometric Dataset for Information Retrieval," International Journal of Electronics Engineering, vol. 11, issue 1, p. 304, Jan. 2019

[16] M. Yasmin, S. Mohsin, I. Irum, and M. Sharif, "Content-based image retrieval by shape, color and relevance feedback," Life Science Journal, vol. 10, pp. 593-598, 2013

[17] N. Bagri and P. K. Johari, "A comparative study on feature extraction using texture and shape for content-based image retrieval," International Journal of Advanced Science and Technology, vol. 80, pp. 41-52, 2015

[18] M. K. Kundu, M. Chowdhury, and S. R. Bulò, "A graph-based relevance feedback mechanism in content-based image retrieval," Knowledge-Based Systems, vol. 73, pp. 254-264, 2015.

[19] M. Singha and K. Hemachandran, "Content-based image retrieval using color and texture," Signal \& Image Processing, vol. 3, p. 39, 2012.

[20] M. Singha and K. Hemachandran, Content-based image retrieval using color and texture," Signal \& Image Processing, vol. 3, p. 39, 2012.

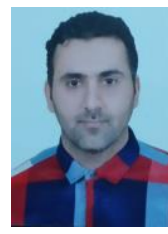

Mudhafar Jalil Jassim Ghrabat is currently an Ph.D student at Huazhong University of Science and Technology, Wuhan, China. He received his master degree in information technology from SRM University, India, Chennai in 2015. His current research interests include credit scoring using machine learning algorithms, network security.

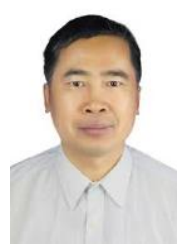

Guangzhi Ma is the associate professor of computer science in Computer School of Huazhong University of Science and Technology (HUST). His research spans data mining, artificial intelligence, and net computing. He gains his master degree and Ph.D. of computer science both in HUST, China.

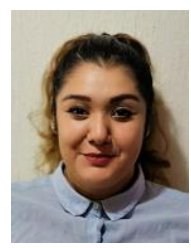

Paula Leticia Pinon Avila received her bachelor degree of engineering science from Instituto Tecnológico de Morelia, Mexica. she received her master degree from the Faculty of Engineering of Huazhong, University of Science and Technology (HUST), China. Her current research interests include using a machine learning algorithm, deep machine learning algorithm, artificial intelligence

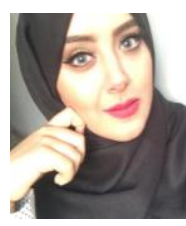

MUNA JALIL JASSIM. She received her bachelor degree in computer science from Alrafidain University College, Iraq, Baghdad2016. Awarded excellence in the Faculty of Rafidain University 2017.she is current research interests include credit scoring using. The machine learning algorithm, Image Processing, development algorithms.

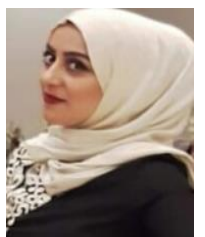

Safa Jalil Jassim received her bachelor degree from Mustansiriya University, Iraq, Baghdad in 2011. She received her master degree from the Faculty of Electrical Engineering, Anna University, India, Chennai in 2016. Her current research interests include credit scoring using machine learning algorithm, big data, control of information systems. 